\title{
Estilos de vida y cronotipo de estudiantes universitarios en Popayán-Colombia
}

\section{Styles of life and chronotype of university students in Popayán-Colombia}

\author{
Nancy-J Molano-T $^{1}$; Luz-M Chalapud-N $^{2}$; Dolly-X Molano- $^{3}$ (D) \\ Forma de citar: Molano NJ, Chalapud LM, Molano DX. Estilos de vida y cronotipo de estudiantes universitarios en Popayán- \\ Colombia. Salud UIS. 2020; 53: e21004. doi: https://doi.org/10.18273/saluduis.53.e:21004 @c) (1)
}

\section{Resumen}

Introducción: la vida cambiante de los universitarios proporciona modificaciones en sus estilos de vida, esto influye en aspectos de salud, al igual que en la relación entre vigilia y sueño por sus actividades académicas, lo que denota una necesidad de ser estudiada, para orientar acciones de prevención de enfermedades a futuro. Objetivo: identificar la asociación entre los estilos de vida y el cronotipo de estudiantes universitarios en Popayán-Colombia. Métodos: estudio cuantitativo de tipo descriptivo correlacional de cohorte transversal, realizado en la ciudad de Popayán-Colombia en una Institución de Educación Superior durante el II periodo de 2017 y I del 2018, con una población de 342 universitarios, quienes cumplieron los criterios de inclusión como estar matriculados durante los periodos establecidos, asistir a las evaluaciones y firmar el consentimiento informado. Mediciones principales: variables antropométricas (peso y talla corporal, perímetro de cintura), presión arterial, cuestionario de estilos de vida PEPS-I, y cuestionario de Matutinidad-Vespertinidad De Horne y Östberg. Resultados: se evidenció moderada asociación entre los estilos de vida y el cronotipo de los estudiantes $(\mathrm{r}=0,593 ; \mathrm{p}=0,000)$, no obstante, la edad y el sexo son variables claves para asociaciones altas para IMC $(\mathrm{r}=0,938 ; \mathrm{p}=0,004)$ y perímetro de cintura $(\mathrm{r}=0858$; $\mathrm{p}=0,000$ ). Conclusión: Los estilos de vida inciden en el cronotipo de los estudiantes, planteándose la necesidad de generar campañas de prevención como de promoción de la salud en esta población.

Palabras clave: Estilos de vida; Cronotipo; Universitarios; Salud.

\begin{abstract}
Introduction: The changing life of university students provides changes in their lifestyles, which influences health aspects, as well as the relationship between wakefulness and sleep due to their academic activities, which denotes a need to be studied, to guide actions of Disease prevention in the future. Objective: To identify the association between lifestyles and the chronotype of university students in Popayán-Colombia. Methods: quantitative descriptive correlational study of a transversal cohort, conducted in the city of Popayán-Colombia in a Higher Education Institution during the II period of 2017 and I of 2018, with a population of 342 university students, who met the inclusion criteria how to be enrolled during the established periods, attend the evaluations and sign the informed consent. Main measurements: anthropometric variables (body weight and height, waist circumference),

1. Universidad del Cauca. Popayán, Colombia.

2. Corporación Universitaria Autónoma del Cauca. Popayán, Colombia.

3. Universidad Santiago de Cali. Valle, Colombia.

Correspondencia: Nancy Janneth Molano Tobar. Dirección: Calle 5 4-70 Popayán, Colombia. Teléfono: +2 8209800 Correo electrónico najamoto@unicauca.edu.co
\end{abstract}


blood pressure, PEPS-I lifestyle questionnaire, and Horne Y Östberg Maturity-Vespertinity questionnaire. Results: there was a moderate association between the lifestyles and the chronotype of the students $(r=0.593 ; \mathrm{p}=0.000)$, however, age and sex are key variables for high associations for BMI $(r=0.938 ; p=0.004)$ and waist circumference $(r=0858 ; p=0.000)$. Conclusions: The lifestyles affect the chronotype of the students, considering the need to generate prevention campaigns as well as health promotion in this population.

Keywords: Lifestyles; Chronotype; University; Health.

\section{Introducción}

Los estilos de vida han sido denominados como "el conjunto de pautas y hábitos comportamentales cotidianos de una persona", los cuales se ven influenciados por la nutrición, las relaciones interpersonales, los cuidados que se realicen en la salud, el ejercicio entre otros; todos ellos se han visto modificados debido a que los patrones y conductas del ser humano han cambiado por diversos procesos ${ }^{2}$.

La Organización Mundial de la Salud (OMS), ha identificado a la población estudiantil universitaria, como una comunidad que afronta grandes cambios relacionados con los estilos de vida ${ }^{3}$, muchos de los jóvenes al ingresar a las universidades cambian de ciudad, esto genera variaciones en su alimentación y rutina; además, los nuevos procesos académicos requieren mayor dedicación y esto conlleva a modificaciones en los horarios de sueño y alimentación. De igual manera, se ha considerado que el estrés es otro indicador de los cambios por los que atraviesan los universitarios, ya que deben enfrentar nuevas relaciones interpersonales y actividades académicas ${ }^{4}$ que los obligan a redimensionar su estilo de vida.

Actualmente se considera que el proceso de sueño y vigilia, es necesario para la salud y su variación puede influir en trastornos metabólicos y hormonales ${ }^{5}$, por ello la importancia de determinar la asociación entre los estilos de vida y el cronotipo de los estudiantes universitarios. La perturbación del sueño es relevante para los procesos de salud, que puede verse alterada si se vincula la intensidad horaria de las clases con el manejo de la carga académica universitaria ${ }^{6}$. A futuro, con estos planteamientos se busca a emprender acciones que permitan generar políticas institucionales y públicas para el manejo del estrés que está afectando a los adolescentes y que indican elevados niveles de depresión, ansiedad e incluso suicidio ${ }^{7}$.

No obstantes, la necesidad de vincular la relación sueño vigilia en los universitarios debe ser una prioritaridad, ya que en algunas publicaciones se indica, como la falta de sueño conlleva a la perturbación en la melatonina ${ }^{8}$, lo que incide con la alteración de los estado de ánimo9 ${ }^{9}$, un déficit de glicemia ${ }^{10}$ como mayor consumo de alimentos procesados y obesidad ${ }^{11}$.

Los estilos de vida inadecuados pueden afectar la salud de la población ${ }^{12,13}$, de ahí la necesidad de plantear una investigación que relacione los estilos de vida con el cronotipo de una población universitaria, que derivaría en la generación de pautas que a futuro incidan en políticas institucionales y gubernamentales que permitan fomentar la salud de esta población ${ }^{14}$. El objetivo que se planteó para la investigación fue identificar la asociación entre los estilos de vida y el cronotipo de estudiantes universitarios en PopayánColombia.

\section{Metodología}

El estudio presentó un enfoque cuantitativo de tipo descriptivo correlacional, con una muestra de 342 estudiantes de una Institución de Educación Superior (IES) Pública de la ciudad de Popayán-Colombia, quienes cumplieron los criterios de inclusión definidos como: estar matriculados en los periodos II de 2017 y I de 2018, asistir a las mediciones y evaluaciones correspondientes y firmar el consentimiento informado, a los menores de 18 años se pidió firma de tutores.

Variables: Pruebas antropométricas, como talla y peso corporal, realizadas por personal calificado y según los estándares y protocolos internacionales de la Sociedad Internacional para el Avance de la Cineantropometría ${ }^{15}$. La masa corporal se determinó teniendo en cuenta que la persona se encontrara con ropa ligera, sin calzado y sin ningún tipo de objetos metálicos o joyas que pudieran interferir en los resultados. El peso corporal se midió en una balanza de impedancia bioeléctrica marca OMRON (modelo HBF-514C), calibrada, con corriente de $50 \mathrm{kHz}$, sin ninguna complicación para las personas evaluadas; la talla se evaluó con un estadiómetro portátil con rango 0-220 cm de $1 \mathrm{~mm}$ de precisión. Se tuvo en cuenta las indicaciones de la OMS para la clasificación del IMC que comprendió los rangos de desnutrición, normal, sobrepeso y obesidad grado I y II ${ }^{16}$. 
Se midió el perímetro de cintura según las indicaciones de la OMS, se determinó el "punto intermedio de la distancia vertical entre el reborde costal inferior y el borde externo medio de la cresta iliaca, en un plano paralelo al suelo"17.

El estilo de vida se evaluó bajo el cuestionario de perfil de estilos de vida (PEPS-I) de Nola Pender (1996) ${ }^{18}$, conformado por 48 ítems tipo Likert, cuyo patrón de respuesta corresponde a cuatro características (nunca, a veces, frecuentemente, rutinariamente), la menor puntuación corresponde a 48 y la mayor a 192, este cuestionario evalúa las dimensiones de nutrición, responsabilidad en salud, manejo del estrés, soporte interpersonal y auto actualización.

Para determinar el cronotipo se utilizó el cuestionario Horne-Ostberg que contiene 19 preguntas, una vez terminado se suman los resultados y de acuerdo con los rangos establecidos se define el tipo de cronotipo presente y éste se clasifica como madrugador, trasnochador o vespertino, intermedio ${ }^{18}$.

Para el análisis estadístico se utilizó el programa Statistical Package for Social Science ${ }^{\circledR}$ software (SPSS versión 24; Chicago, IL, USA) para Windows, para identificar la normalidad de la muestra se utilizó el estadístico de Kolgomoriv-Smirnov. Se aplicaron métodos estadísticos básicos, los valores continuos se expresaron como media y $( \pm)$ desviación estándar y las proporciones en porcentaje, se realizó análisis de varianza (ANOVA) para estudiar las diferencias de cronotipo; análisis correlacionales mediante el coeficiente de correlación de Pearson considerando un nivel de significancia de $\mathrm{p}<0.05$.

\section{Resultados}

Los resultados mostraron una distribución normal de la población frente a las variables de estudio, lo cual se obtuvo con la aplicación de la prueba de normalidad de Kolgomrov- Smirnov.

La población muestra del proyecto correspondió a 342 universitarios, distribuidos en 182 mujeres (53,2\%) y 160 hombres (46,8\%), con una edad promedio de

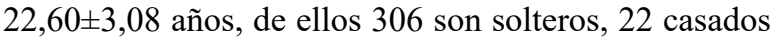
y 13 en unión libre.

Se evaluó el departamento de procedencia de los estudiantes, se evidenció que 232 estudiantes son del departamento del Cauca (67,8 \%), seguido del departamento de Nariño (20,8 \%) y Valle del Cauca con una proporción menor de 4,1\%. El estrato socioeconómico de los estudiantes se ubica con mayor proporción en el estrato 2 con $47,1 \%$ y estrato 3 representado en un $24 \%$.

Con relación a las variables antropométricas se estableció que los estudiantes presentaron un índice de masa corporal (IMC) normal (52,6 \%) seguido de sobrepeso en 145 estudiantes ( $42,4 \%)$; al estratificar por sexo se evidenció que las mujeres presentaron mayores valores en normal $(\mathrm{n}=92)$ y sobrepeso $(\mathrm{n}=79)$ que los hombres, los cuales se distribuyeron en los rangos de normal $(\mathrm{n}=88)$ y sobrepeso $(\mathrm{n}=66)$.

Para el perímetro de cintura se determinó, que la población presentó riesgo bajo $76,6 \%$ y riesgo moderado en $17,8 \%$, al estimar la variable según el sexo se notó una significancia estadística $(p<0,0001)$, donde las mujeres presentaron valores superiores en la clasificación de riesgo bajo $(\mathrm{n}=170)$ y en riesgo alto $(n=10)$, al de los hombres que en riesgo bajo fue de $n=92$ y para riesgo alto solo $\mathrm{n}=9$, mientras que en el ítem de riesgo moderado se presentó un mayor indicador para los hombres $(n=59)$ que para las mujeres $(n=2)$.

De los estilos de vida se puede indicar que los ítems relacionados con auto actualización, como las relaciones interpersonales, tienen mayor relevancia para los universitarios, caso opuesto al encontrado con el aspecto de salud, donde los estudiantes presentaron menor interés, como se observa en la Gráfica 1.

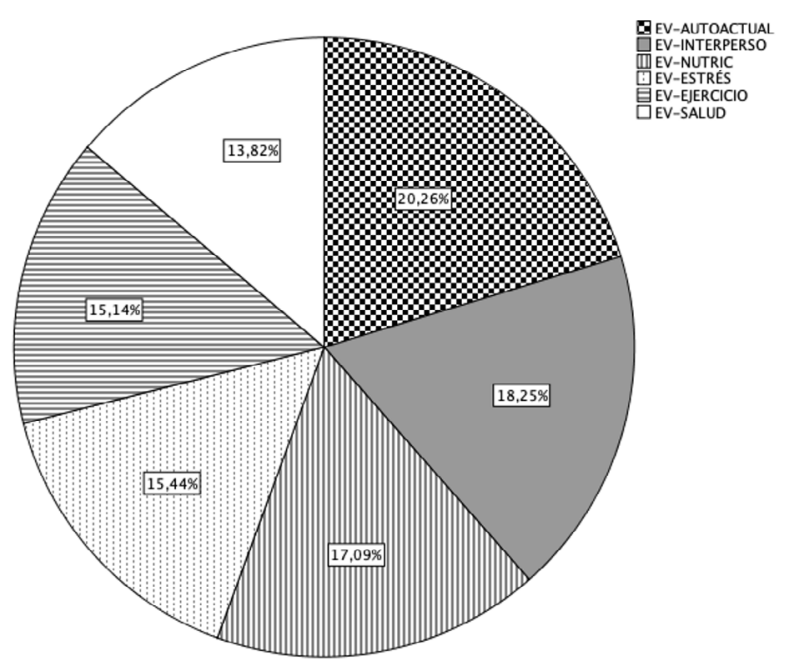

Gráfica 1. Distribución de los Estilos de vida en la población.

En la Tabla 1, se aprecia el promedio y desviación estándar de las dimensiones del perfil de estilos de vida, lo que indica que la población presento una significancia estadística para estos parámetros evaluados. 
Respecto al cronotipo, se pudo determinar que el 66,1\% de la población presenta un cronotipo intermedio, seguido de matutino moderado (27,2\%). Al asociarlo de acuerdo al sexo, se encontró la misma tendencia, donde 123 mujeres presentaron cronotipo intermedio y 52 matutino moderado, para el caso de los hombres se clasificaron en intermedio 103 y 41 hombres cronotipo matutino moderado.

Tabla 1. Perfil de estilos de vida según el sexo.

\begin{tabular}{lccc}
\hline \multicolumn{1}{c}{ Variable } & $\begin{array}{c}\text { Femenino } \\
\text { (Media } \pm \text { Desviación estándar) }\end{array}$ & $\begin{array}{c}\text { Masculino } \\
\text { (Media } \pm \text { Desviación estándar) }\end{array}$ & $\begin{array}{c}\text { Chi Cuadrado } \\
(\mathbf{p}<\mathbf{0 , 0 5})\end{array}$ \\
\hline Nutrición & $2,76 \pm 0,634$ & $2,91 \pm 0,686$ & 0,000 \\
Ejercicio Físico & $2,21 \pm 0,794$ & $2,73 \pm 0,752$ & 0,000 \\
Salud & $2,38 \pm 0,590$ & $2,27 \pm 0,716$ & 0,000 \\
Estrés & $2,54 \pm 0,740$ & $2,49 \pm 0,700$ & 0,000 \\
Relaciones Interpersonales & $3,07 \pm 0,698$ & $2,89 \pm 0,630$ & 0,000 \\
Auto Actualización & $3,31 \pm 0,570$ & $3,31 \pm 0,585$ & 0,000 \\
\hline
\end{tabular}

$\mathrm{Al}$ analizar el coeficiente de correlación de Pearson, se identificaron relaciones con indicadores muy altos, altos y moderados como se observa en la Tabla 2, las demás relaciones se encontraron bajas o nulas, por lo tanto, no se presentan. Lo anterior indica que el sexo presenta una correlación positiva para las variables relacionadas con parámetros antropométricos como dimensiones de nutrición y relaciones interpersonales del perfil de estilos de vida, se aprecia una correlación significativa entre los estilos de vida y el cronotipo en la población de estudiantes universitarios.

Tabla 2. Correlación de variables.

\begin{tabular}{lccc}
\hline \multicolumn{1}{c}{ Variables } & Valor R & Valor P & Correlación \\
\hline Sexo/IMC & 0,938 & 0,004 & Muy alta \\
Sexo/perímetro de cintura & 0,858 & 0,000 & Muy alta \\
Estilos de vida/Edad & 0,668 & 0,013 & Alta \\
Cronotipo/Estilos de vida & 0,593 & 0,000 & Moderada \\
Sexo/EV nutrición & 0,566 & 0,000 & Moderada \\
Sexo/EV Relaciones interpersonales & 0,421 & 0,009 & Moderada \\
\hline
\end{tabular}

\section{Discusión}

Los resultados de la población universitaria indican que gran parte de esta se concentra en la misma región que cubre la universidad, y por ser una IES pública permite que la cobertura se genere en estratos socioeconómicos 2 y 3 , esto facilita la educación superior a una comunidad con ingresos económicos de bajos a moderados y con ciertas necesidades básicas de vida satisfechas como son servicios públicos y transporte. Diversos estudios establecen que una de las misiones de las Instituciones de Educación Superior, en especial las públicas, es ofrecer educación a población de estratos bajos y medios ${ }^{19,20}$, lo cual contribuye a que un país se cualifique y progrese.

Es indudable que los estudiantes universitarios se ven expuestos a numerosas acciones que inciden en la presencia de sobrepeso u obesidad ${ }^{21}$, lo que para la población en estudio se evidenció, es que en ellos no infieren dichas apreciaciones, pero sí que el $42,4 \%$ de ellos presentaron sobrepeso, esta situación como se ha dicho con anterioridad, se encontró en la investigación con estudiantes universitarios de la ciudad de Pamplona-Colombia ${ }^{22}$, pero difieren a nivel internacional donde la prevalencia es hacia el sobrepeso como tal y a la obesidad en sus diferentes $\operatorname{grados}^{23}$, quizás se presente esta disparidad entre las poblaciones debido a que Colombia goza de una variedad alimentaria y una ciudad como Popayán, por ser pequeña y de fácil accesibilidad, permita mantener una alimentación dentro de los rangos adecuados, hecho que merece investigarse desde otra dimensión.

Se logró establecer que el IMC presentó mayor proporción en las mujeres, esto es similar a otros estudios tanto nacionales como internacionales, explicado desde los procesos hormonales que conllevan a que se propicie mayor adiposidad ${ }^{24}$, esto en 
concordancia aspectos filogenéticos donde las mujeres tienden a acumular mayor grasa que sus homólogos ya que es una medida de preparación a procesos gestacionales futuros, de igual forma se logró establecer una correlación muy alta entre IMC y el sexo, como se evidencia en otros estudios poblacionales puesto que las mujeres tienden a generar mayor consumo de alimentos ricos en azucares ${ }^{25}$, asociado al estrés que manejan en ciertas ocasiones, lo que conlleva a incrementar su peso y medidas corporales.

Acerca del perímetro de cintura, en la población general se evidenció que la prevalencia fue de bajo riesgo. Cabe notar que el riesgo moderado predomina en los hombres, lo que se asocia con los estudios nacionales ${ }^{26,27}$ e internacionale ${ }^{28}$ donde se confirma que el perímetro de cintura es un indicador importante para determinar el riesgo cardiovascular, por su asociación con la grasa visceral ${ }^{29}$. Éste es considerado un órgano endocrino importante al cual deberá realizarse un seguimiento y vincularlo a futuro con otros parámetros bioquímicos; teniendo en cuenta que los hombres tienen una distribución de la grasa corporal con predominio en el área abdominal, esto propicia un estado de inflamación que conlleva a un estrés oxidativo, disfunción endotelial $\mathrm{y}$ ateroesclerosis ${ }^{30}$.

El estudio permitió determinar que los estudiantes universitarios tienen estilos de vida poco saludables, y se correlacionan con la edad; esto coincide con los aportes de Maganto, et al. ${ }^{31}$, que infieren que los jóvenes adoptan estos poco o nada saludables hábitos debido a la poca estabilidad psicológica, social y emocional. En cuanto al parámetro de nutrición, asociado con el sexo, se comprobó que éste es moderado, ello es explicado por Celis, et al. ${ }^{32}$, al mencionar que los alimentos que los jóvenes suelen consumir son escogidos por su rápida preparación y no por su contenido nutricional, lo que para ellos se asocia a las comidas chatarra ${ }^{33}$, esto se manifiesta con comportamientos alimentarios anormales mediados por factores como crisis depresivas, el estrés y la ansiedad ${ }^{34}$.

Otra asociación moderada encontrada en el estudio correspondió a las relaciones interpersonales, las cuales también se vieron afectadas en otros estudios ${ }^{35}$, y es explicado por Shu-Chu y Wei-Chu ${ }^{36}$ quienes aluden que la tecnología ha influido notablemente en las relaciones interpersonales, pues el uso de las redes sociales ha alejado a las personas de sus entornos inmediatos ${ }^{37}$, lo que afecta el comportamiento y las mismas relaciones entre sus pares de sexo opuesto, lo que puede incidir en la salud ${ }^{38}$, pues es mayor el tiempo que pasan frente al computador que el que dedican a salir a pasear o divertirse en persona ${ }^{39}$.

El cronotipo presente en los estudiantes correspondió a un tipo intermedio, lo que se relaciona con otros estudios $^{40}$, donde el mayor porcentaje es de tipo intermedio debido a que el horario de sueño de los estudiantes varía entre lunes - viernes y los fines de semana. El otro ítem prevalente es el matutino, que se explica debido a que la mayoría de su carga académica es a tempranas horas de la mañana, y esto genera dicha propensión. Estos hechos contradicen otros estudios donde se ha determinado que los estudiantes universitarios tienen tendencias predominantes de noctámbulos, debido a las actividades asociadas al ocio y manejo de su tiempo libre ${ }^{41}$ que realizan en la noche.

Se pudo confirmar la hipótesis sobre la asociación entre los estilos de vida y el cronotipo, que para este estudio fue moderada, similar a lo encontrado en otros estudios ${ }^{42}$. Se determina que el déficit de sueño es afectado por el estilo de vida y hábitos de los estudiantes universitarios ${ }^{43}$, se recalca la importancia de los estilos de vida saludables para una buena relación entre sueño y vigilia, situación de relevancia si nos detenemos a pensar que la mayoría de los estudiantes universitarios se encuentran en una etapa de desarrollo de sus capacidades tanto físicas como intelectuales.

\section{Conclusiones}

Se logró establecer que los jóvenes universitarios presentaron estilos de vida poco saludables al relacionarse con los aspectos de la salud y el ejercicio físico, lo cual indica la necesidad de incluir dentro de los entornos universitarios medidas que fomente conductas hacia la salud y la actividad física.

Referente al cronotipo se apreció que la tendencia de los universitarios se centra en una relación sueño vigilia intermedia, hecho que podría variar por la vinculación de otras conductas negativas y generar problemas con el equilibrio en el ritmo circadiano.

La población estudiantil presentó una asociación moderada entre el cronotipo y los estilos de vida poco saludables, lo que indica una situación de riesgo a futuro, por lo tanto, la implementación de acciones preventivas desde la institución como de la población, podría disminuir el riesgo de enfermedad. 
El sexo y la edad son parámetros determinantes para el sobrepeso y la obesidad, considerando necesario un seguimiento relacionado a su nivel de actividad física como hábitos de vida, de la misma manera contrastar las mediciones presentadas con otros factores bioquímicos, podrá permitir mayores alcances en cuanto a su estado de salud.

\section{Consideraciones éticas}

La investigación estuvo avalada por el comité de ética de la Universidad del Cauca bajo el ID4675, teniendo en cuenta la declaración de Helsinky para la investigación con seres humanos como la resolución 8439 de 1990 del Ministerios de Salud y Protección Social de Colombia, todos los participantes asistieron y firmaron el consentimiento informado.

\section{Conflicto de interés}

Los autores manifiestan no presentar conflictos de interés y haber participado en la concepción, diseño y demás actividades relacionadas con la publicación del articulo.

\section{Referencias}

1. Sanchez-Ojeda MA, De Luna-Bertos E. Hábitos de vida saludable en la población universitaria. Nutr Hosp. 2015; 31(5): 1910-1919. doi: http://dx.doi. org/10.3305/nh.2015.31.5.8608

2. Valandia Galvis ML, Arenas Parra JC, Ortega Ortega N. Estilos de vida en los estudiantes de enfermería. Rev Cienc Cuid. 2015; 12(1): 27-39.

3. Rodríguez F, Espinoza Oteiza L, Galvez Carvajal J, Macmillan Kuthe P. Estado nutricional y estilos de vida en estudiantes universitarios de la Pontificia Universidad Católica de Valparaíso. Univ Salud. 2013; 15(2): 123-135.

4. Rizo-Baeza M, González-Brauer N, Cortés E. Calidad de la dieta y estilos de vida en estudiantes de Ciencias de la Salud. Nutr Hosp. 2014; 29(1): 153-157. doi: http://dx.doi.org/10.3305/ nh.2014.29.1.6761

5. Machado A, Díaz I, De la Torre ME. Un breve acercamiento al cronotipo humano. Medicentro Electrónica. 2018; 22(1): 74-76.

6. Gallardo-Solarte K, Benavides-Acosta FP, RosalesJiménez R. Costos de la enfermedad crónica no transmisible: la realidad Colombiana. Rev Cienc Salud. 2016; 14(1): 103-114. doi: dx.doi. org/10.12804/revsalud14.01.2016.09
7. Hernández-Cortés LM, Londoño Pérez C. Imagen corporal, IMC, afrontamiento, depresión y riesgo de TCA en jóvenes universitarios. An Psicol. 2013; 29(3): 748-761. doi: http://dx.doi.org/10.6018/ analesps.29.3.175711

8. Montaruli A, Galasso L, Caumo A, Cè E, Pesenti C, Roveda E, et al. The circadian typology: the role of physical activity and melatonin. Sport Sci Health. 2017; 13(3): 469-76. doi: https://doi.org/10.1007/ s11332-017-0389-y

9. Garjón J. Melatonina para los trastornos del sueño. Bol Inf Farmacoter Navarra. 2014; 22(1): 1-12.

10. Guo Y, Liu Y, Huang X, Rong Y, He M, Wang Y, et al. The effects of shift work on sleeping quality, hypertension and diabetes in retired workers. PLoS One. 2013; 8(8): 1-7. doi: https://doi.org/10.1371/ journal.pone.0071107

11. Díaz-Rodríguez E, Garnacho-Gayarre N, ValdéCañedo M, Marín-Fernández B, Díaz-Lopez B. Efectos de la melatonina sobre el mantenimiento de parámetros corporales durante el envejecimiento. Estudio en Rattus norvegicus. Rev Esp Geriatr Gerontol. 2001; 36(5): 287-292. doi: 10.1016/ S0211-139X(01)74738-5

12. Martínez MD, Miguel-Soca P, Rodríguez R, Lopez Baster J, Ponce de León D. Prevalencia de obesidad abdominal y factores de riesgo cardiovascular asociados en adultos jóvenes. Rev Cuba Salud Pública. 2017; 43(3): 1-17.

13. Campo Y, Pombo LM, Teherán AA. Estilos de vida saludable y conductas de riesgo en estudiantes de medicina. Rev Univ Ind Santander Salud. 2016; 48(3): 301-309. doi: https://doi.org/10.18273/ revsal.v48n3-2016004

14. Aliaga-Díaz E, Cuba-Fuentes S, Mar-Meza M. Promoción de la salud y prevención de las enfermedades para un envejecimiento activo y con calidad de vida. Rev Peru Med Exp Salud Publica. 2016; 33(2): 311. doi: https://doi.org/10.17843/ rpmesp.2016.332.2143

15. Pérez BM, Landaeta-Jiménez M, Arroyo Barahona E, Marrodán MD. Patrón de actividad física, composición corporal y distribución de la adiposidad en adolescentes venezolanos. Rev An Venez Nutr. 2012; 25(1): 5-15.

16. Ministerio de Salud y Protección Social. Resolución 2465 de 2016. 14 jun 2016; p. 1-47.

17. Martín-Castellanos Á, Armesilla MDC, BarcaDurán FJ, Castellanos PM, Gómez JJ. Obesidad y riesgo de infarto de miocardio en una muestra de varones europeos. El índice cintura-cadera sesga el riesgo real de la obesidad abdominal. Nutr Hosp. 2016; 33(5): 1108-15. doi: http://dx.doi. org/10.20960/nh.574 
18. Aristizábal-Hoyos GP, Blanco-Borjas DM, Sánchez-Ramos A, Ostiguín Melendez RM. El modelo de promocion de la Salud de Nola Pender. Una reflexión en torno a su comprensión. Enferm Univ. 2011; 8(4): 16-23.

19. Valladares M, Campos B, Zapata C, Durán Agüero $\mathrm{S}$, Obregón AM. Asociación entre cronotipo $\mathrm{y}$ obesidad en jóvenes. Nutr Hosp. 2016; 33(6): 13361339. doi: https://doi.org/10.20960/nh.792

20. Ministerio de Educación Nacional Documento $n^{\circ} 20$. Documento $n^{\circ} 20$. Sentido de la educación inicial. 2014.

21. Uzcátegui Montes K, Cabrera de los Santos B, Lami P. The Inclusive Education: a Way for Integration. Divers Perspect en Psicol. 2012; 8(1): 139-150.

22. Cuevas-Nasu L, Rivera-Dommarco JÁ, GómezAcosta LM, Morales-Rúan MDC, Shamah-Levy T, Hernández-Ávila M, et al. Sobrepeso y obesidad en niños y adolescentes en México, actualización de la Encuesta Nacional de Salud y Nutrición de Medio Camino 2016. Salud Publica Mex. 2018; 60(3): 244. doi: https://doi.org/10.21149/8815

23. Tuta-García H, Lee-Osomo B, Matinez-Torres Ja. Prevalencia y factores asociados a sobrepeso y obesidad, en estudiantes universitarios de 18 a 25 años, en Pamplona Norte de Santander durante el primer periodo del 2013. Ces Salud Publica. 2015; 19-24.

24. Mollinedo F, Trejo P, Araujo R, Lugo L. Índice de masa corporal, frecuencia y motivos de estudiantes universitarios para realizar actividad física. Rev Cuba Educ Medica Super. 2013; 27(3): 189-199.

25. Múnera Gaviria HA, Salazar Blandón DA, Pastor Durango MP, Alzate Yepes T. Overweight and obesity conditions: prevalence and associated risk factors in nursing students in a public university in Medellín, Colombia. Invest Educ Enferm. 2017; 35(2): 191-198. doi: https://doi.org/10.17533/udea. iee.v35n2a08

26. Cabezas Zabala CC, Hernández Torres BC, Vargas Zárate M. Azúcares adicionados a los alimentos: efectos en la salud y regulación mundial. Revisión de la literatura. Rev la Fac Med. 2016; 64(2): 319. doi: https://doi.org/10.15446/revfacmed.v64n2.52143

27. Ramírez-Vélez R, Triana-Reina HR, Carrillo HA, Ramos-Sepúlveda JA. Percepción de barreras para la práctica de la actividad física y obesidad abdominal en universitarios de Colombia. Nutr Hosp. 2016; 33(6): 1317-1323. doi: https://doi. org/10.20960/nh.777

28. Martín-Castellanos Á, CabañasArmesilla MD, BarcaDurán FJ, Castellanos PM, Gómez Barrado JJ. Obesidad y riesgo de infarto de miocardio en una muestra de varones europeos. El índice cinturacadera sesga el riesgo real de la obesidad abdominal. Nutr Hosp. 2016; 33(4): 88-95. doi: http://dx.doi. org/10.20960/nh.982

29. Wennman H, Kronholm E, Partonen T, Tolvanen A, Peltonen M, Vasankari $\mathrm{T}$, et al. Interrelationships of Physical Activity and Sleep with Cardiovascular Risk Factors: a Person-Oriented Approach. Int J Behav Med. 2015; 22(6): 735-747. doi: https://doi. org/10.1007/s12529-015-9470-6

30. Chen YC, Lai SH, Tsai YW, Chang SS. Visceral Adiposity Index as a Predictor of chronic kidney disease in a relatively healthy population in Taiwan. J Ren Nutr. 2018; 28(2): 91-100. doi: https://doi. org/10.1053/j.jrn.2017.07.006

31. Maganto C, Garaigordobil M, Kortabarria YL. Variables antropométricas, hábitos $\mathrm{y}$ dietas alimentarias en adolescentes y jóvenes: diferencias en función del sexo. Acción Psicológica. 2016; 13(2): 89-100. doi: http://dx.doi.org/10.5944/ ap.13.2.17817

32. Celis G, Obregón MC, Ayala J, Blackburn N, Gómez M, Páez V, et al. La obesidad y su relación con otros factores de riesgo cardiovascular en una población de estudiantes universitarios del centro de Bogotá, D.C. NOVA. 2013; 11(20): 35-38.

33. Becerra S. Descripción de las conductas de salud en un grupo de estudiantes universitarios de Lima. Rev Psicol. 2016; 34(2): 239-260. doi: https://doi. org/10.18800/psico.201602.001

34. Cerezo Correa P, Vergara Quintero MC, Nieto Murillo E, Cifuentes Aguirre OL, Parra Sánchez JH. Caracteristicas de salud publica de estudiantes de una universidad privada de la ciudad de Manizales. Rev Hacia Prom Salud. 2011; 16(1): 73-86.

35. Obregón Soto MP. Phubbing y las relaciones interpersonales en adolescentes. Rev Medios y Educ. 2015; 50: 173-185.

36. Shu-Chu SL, Wei-Chun H. Lifestyles, innovation attributes, and teachers' adoption of game-based learning: Comparing non-adopters with early adopters, adopters and likely adopters in Taiwan. Comput Educ. 2016; 96: 29-41. doi: https://doi. org/10.1016/j.compedu.2016.02.009

37. Fajardo Bonilla E, Angel Aragon LA. Prevalencia de sobrepeso y obesidad, consumo de alimentos y patron de actividad fisica en una poblacion de niños escolares de la ciudad de Bogota. Rev Med. 2012; 20(1): 101-116.

38. Grey M. Lifestyle determinants of health: Isn't it all about genetics and environment? Nurs Outlook. 2017; 65(5): 501-505. doi: https://doi.org/10.1016/j. outlook.2017.04.011 
39. Garay Ruiz U, Tejada Garitano E, RomeroAndonegi A. Rendimiento y satisfaccion de estudiantes universitarios en una comunidad en linea de practicas. Rev Mex Investig Educ. 2017; 22(75): 1239-1256.

40. Pérez-Olmos I, Talero-Gutiérrez C, González-Reyes RB, Moreno C. Ritmos circadianos de sueño y rendimiento académico en estudiantes de medicina. Rev Cienc Salud Bogotá. 2006; 4(1): 147-157.

41. Giménez Badia S, Albares Tendero J, Canet Sanz T, Jurado M. J, Madrid-Pérez JA, Merino Andreu M, et al. Trastorno de retraso de la fase del sueño y del despertar. Síndrome de retraso de fase. Rev Pediatr Aten Primaria. 2016; 18: 129-139.

42. Kabrita CS, Hajjar-Muça TA, Duffy JF. Predictors of poor sleep quality among Lebanese university students: Association between evening typology, lifestyle behaviors, and sleep habits. Nat Sci Sleep. 2014; 6: 11-18. doi: https://doi.org/10.2147/NSS. $\underline{\mathrm{S} 55538}$

43. Vera B, Dashti HS, Gómez-Abellán P, HernándezMartínez AM, Esteban A, Scheer FAJL, et al. Modifiable lifestyle behaviors, but not a genetic risk score, associate with metabolic syndrome in evening chronotypes. Sci Rep. 2018; 8(1): 1-11. doi: https:// doi.org/10.1038/s41598-017-18268-Z 